Article

\title{
Anomalous Magnetization Enhancement and Frustration in the Internal Magnetic Order on $\left(\mathrm{Fe}_{0.69} \mathrm{Co}_{0.31}\right) \mathrm{B}_{0.4}$ Nanoparticles
}

Belén Molina-Concha ${ }^{1, \dagger}$, Roberto D. Zysler ${ }^{1, *}$ and Héctor Romero ${ }^{2}$

1 Centro Atómico Bariloche, CNEA-CONICET, 8400 S.C. de Bariloche, RN, Argentina; E-Mail: molinacb@gmail.com

2 Facultad de Ciencias, Departamento de Física, Universidad de Los Andes, Mérida, 5101, Venezuela; E-Mail: hectorom@ula.ve

$\dagger$ Present address: Intitut National de l'Energie Solaire-Commissariat à l'Energie Atomique, 50 avenue du lac Léman, 73375 Le Bourget du Lac, France

* Author to whom correspondence should be addressed; E-Mail: zysler@cab.cnea.gov.ar; Tel.: +54-294-444-5158; Fax: +54-294-444-5299.

Received: 14 February 2012; in revised form: 19 March 2012 / Accepted: 23 March 2012 / Published: 2 April 2012

Abstract: We have studied the internal magnetic order of 3-nm $\left(\mathrm{Fe}_{0.69} \mathrm{Co}_{0.31}\right)_{0.6} \mathrm{~B}_{0.4}$ amorphous nanoparticles. These nanoparticles were dispersed in a non-magnetic matrix (non-interacting nanoparticles) to contrast them with the powder samples, where strong interparticle interactions are present. In similar fashion to the bulk alloy, this system exhibits a saturation magnetization maximum as a function of Fe composition near 69 at $\%$ Fe for the powder and dispersed samples at all temperatures. The saturation magnetization $\left(M_{S}\right)$ of the dispersed sample shows anomalous behavior, revealing frustration in the internal magnetic order of the particles. Unexpectedly, the $M_{S}$ of the non-interacting sample at low temperatures is larger than the corresponding bulk alloy or the calculated value of $M_{S}$ for the same Fe-Co composition. By contrast, the powder sample has low $M_{S}$ values and it is approximately constant in temperature.

Keywords: fine-particle systems; amorphous magnetic materials; Fe-Co-B alloys; magnetic anisotropy; magnetization enhancement 


\section{Introduction}

Fe-Co alloys have attracted theoretical and technological interest due to their magnetic features. The atomic magnetic moment of the $\mathrm{Fe}_{\mathrm{x}} \mathrm{Co}_{1-\mathrm{x}}$ alloys shows variations with composition following the known Slater-Pauling curve [1], which presents a maximum at $x=0.65$ with a magnetic moment $\mu \sim 0.45 \mu_{\mathrm{B}}$ per atom $(\sim 240 \mathrm{emu} / \mathrm{g})$, at constant temperature. This magnetization is larger than pure metallic Fe or $\mathrm{Co}$, and this behavior is determined by its electronic band structure, i.e., the average concentration of the $3 \mathrm{~d}$ electrons in the alloy.

On the other hand, magnetic nanoclusters have received much attention since the effect of nanoscale confinement leads to a different magnetic behavior from the bulk $[2,3]$. These effects lead to novel properties and possibly new technological applications. It is known that as the particle size decreases, a finite-size effect dominates the magnetic properties due to surface effects. It is expected that the decrease in the coordination number induces a weakening of the exchange interactions between surface atoms. In this case, the picture of a magnetic single-domain particle where all spins are pointing in the same direction, thus leading to a coherent relaxation process, is no longer valid if one considers the effect on the global magnetic properties of the particle of misaligned spins on the surface [4]. Some experiments have shown that the hysteresis loops of very small nanoparticles display high irreversibility fields and do not saturate [5-7] even at high magnetic fields, which indicates that the anisotropy fields cannot be the only responsible mechanism for the magnetization reversal. Usually, these phenomena can be ascribed to intrinsic properties of the particle itself, i.e., spin-glass state of the surface which creates an exchange field on the core of the particle [5,7] or a collective behavior induced by interparticle interactions [8-10], and has been the object of controversy [11]. Some core-shell models have been developed to describe the behavior of these nanoparticles [12-19]. In metallic nanoparticles also, the band structure must be modified by the finite size leading to a confinement of the conduction electron band [20]. These confinement and surface effects can modify the behavior of the Fe-Co alloys, developing the effective magnetic moment and shifting the alloy composition where the maximum of the magnetization is observed. The presence of magnetic interactions between particles can also modify the surface configuration of spins, and the problem becomes more complex.

Previous studies on $\left(\mathrm{Fe}_{\mathrm{x}} \mathrm{Co}_{1-\mathrm{x}}\right)_{0.6} \mathrm{~B}_{0.4}$ nanoparticles show a sharp maximum of the saturation magnetization at room temperature as a function of composition at $x=0.69$ against the broad maximum present in the bulk materials [21]. In this work, we present magnetization results on $\left(\mathrm{Fe}_{0.69} \mathrm{Co}_{0.31}\right)_{0.6} \mathrm{~B}_{0.4}$ nanoparticles, where confinement effects contribute significantly to the magnetic properties of these samples.

\section{Experimental Section}

Amorphous nanoparticles of Fe-Co-B alloy were obtained following a chemical route by reduction of aqueous solutions of metallic salts [22,23]. The relative $\mathrm{Fe} / \mathrm{Co}$ composition was determined by energy dispersive spectroscopy microanalysis (EDX) and the boron concentration by atomic absorption analysis. The iron-cobalt ratio results are close to the nominal one, and the boron concentration was 40 at $\%$. The x-ray powder diffraction patterns show a broad spectrum confirming 
the amorphous nature of the particles. The mean diameter and the particle size were determined by TEM and light scattering experiments [23] giving, for all samples, a lognormal distribution centered at $2.8 \mathrm{~nm}$ with $\sigma=0.3$ (Figure 1). In order to study the magnetic properties of non-interacting particles (or negligible interparticle interactions), the particles were dispersed in a non-magnetic matrix (polyvinyl-pyrrolidone) at about $1 \% \mathrm{w} / \mathrm{w}$. The dispersed samples were produced by adding a colloidal aqueous suspension of the particles (at the end of the synthesis process, before drying the sample) in the polymer solution, preventing the agglomeration of the particles in the matrix. From the particle concentration in the polymer and the particle size, an average interparticle distance of $19 \mathrm{~nm}$ was calculated. Powder samples of the particles were studied in order to observe the interparticle interaction effects. These samples are compacted using a sample holder that presses the powder in order to avoid any movement while measuring magnetization.

Figure 1. Size distribution of the $\left(\mathrm{Fe}_{0.69} \mathrm{Co}_{0.31}\right) \mathrm{B}_{0.4}$ nanoparticles measured by light scattering method (bars). The solid line corresponds to the least-square fitting of the data with a log-normal distribution.

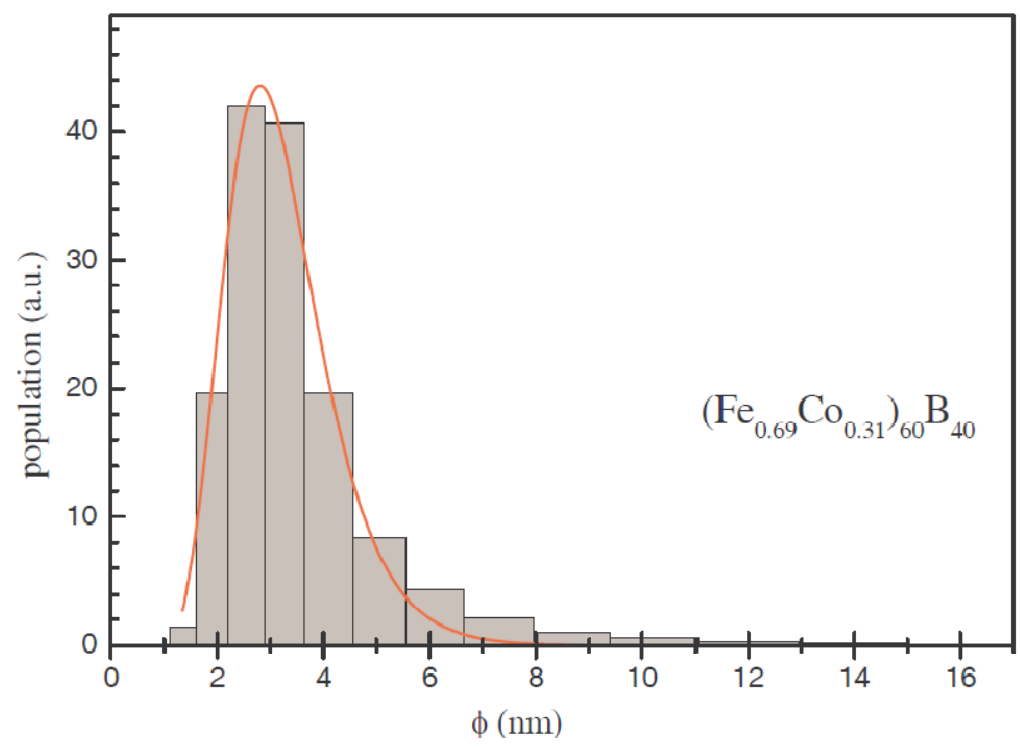

Bulk samples of composition $\left(\mathrm{Fe}_{0.7} \mathrm{Co}_{0.3}\right)_{0.8} \mathrm{~B}_{0.2}$ and $\mathrm{Fe}_{0.7} \mathrm{Co}_{0.3}$ were produced in an arc furnace to contrast their magnetic properties with nanoparticles in the same Fe/Co composition ratio. These samples were also characterized with EDX analysis obtaining the nominal Fe/Co ratio within 2 at $\%$ of error. The boron composition was determined with 7 at $\%$ of error by weighting of the sample.

Magnetization measurements were performed as a function of the magnetic field up to $10 \mathrm{kOe}$ at different temperatures in a commercial VSM, and magnetization measurements as a function of temperature were performed by using a commercial SQUID magnetometer.

\section{Results and Discussion}

As our nanoparticles are ferromagnetically ordered at temperatures above room temperature, single domain behavior is expected to be observed. The magnetization results as a function of temperature of $\left(\mathrm{Fe}_{0.69} \mathrm{Co}_{0.31}\right) \mathrm{B}_{0.4}$ particles measured under zero field cooling (ZFC) and field cooling (FC) conditions applying a field $H=50$ Oe are plotted in Figure 2 for the dispersed and powder samples. 
Figure 2. Magnetization vs. temperature curves for $\left(\mathrm{Fe}_{0.69} \mathrm{Co}_{0.31}\right) \mathrm{B}_{0.4}$ dispersed (a) and powder (b) particles measured under zero-field-cooling (ZFC) and field-cooling (FC) conditions.

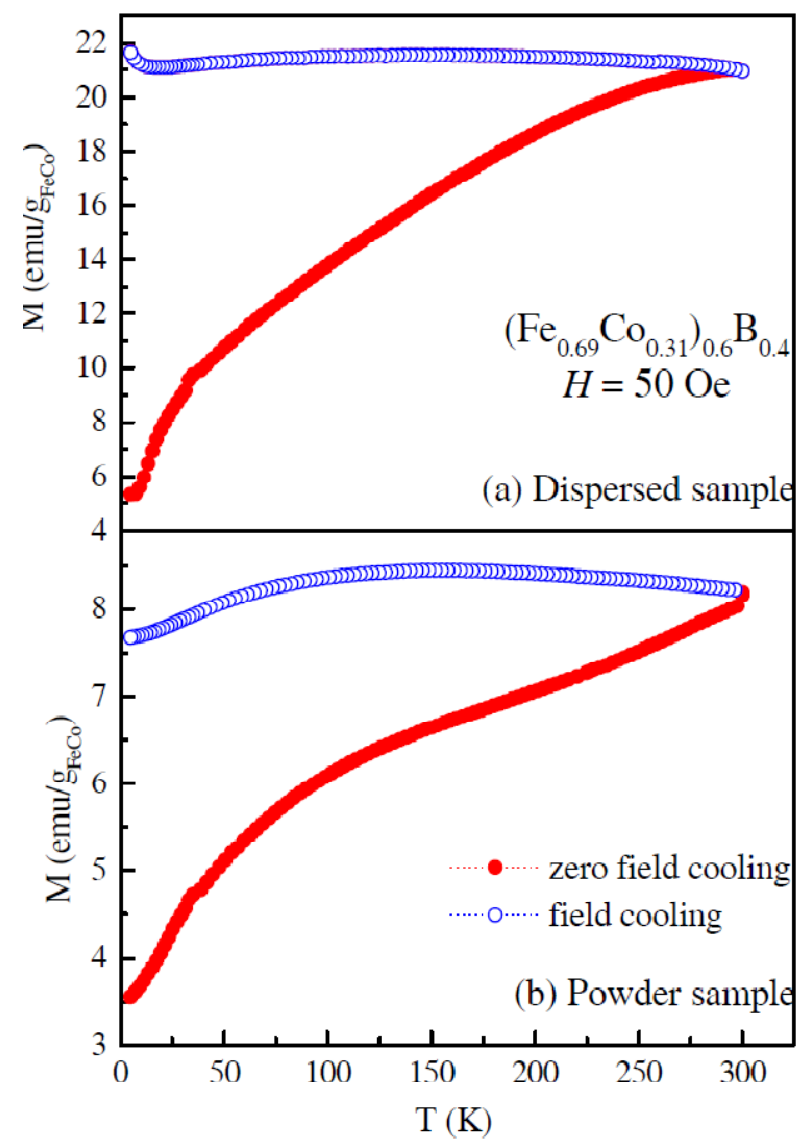

In both systems, the observed behavior represents irreversibility, and consequently the blocking temperature (the temperature above which the system behaves as a superparamagnet) is above room temperature. This blocking temperature is unexpected because the usual energy barrier for $3 \mathrm{~nm}$ amorphous ferromagnetic nanoparticles is small, generally giving a superparamagnetic regime at room temperature. In our case, the coupling of the single domain magnetization of the core of the particle with the surface anisotropy of the surface spins via exchange interaction enhances the effective anisotropy. Taking into account the non-interacting nature of the particles, the FC magnetization curve presents an atypical shape, remaining quasi-constant with decreasing temperature (Figure 2(a)). This fact is probably evidence of intraparticle interactions or magnetic order frustration at the particle surface [16,17]. Monte Carlo simulations of non-interacting Fe-Co nanoparticles within the core-shell model satisfactorily reproduce the measured ZFC-FC magnetization curves [24]. In this model, a different orientation of the uniaxial surface anisotropy axes for $\mathrm{Fe}$ and $\mathrm{Co}$ magnetic moments is considered, producing frustration in the superficial magnetic order. This effect leads to the anomalous FC curve measured on our non-interacting nanoparticles. The increase of the magnetization at low temperatures is also reproduced and it is related to an improvement of the internal magnetic order of the particles, as it will be described later with the hysteresis loops results. On the other hand, the $M(T)$ curve under FC condition of the powder sample, where strong interparticle interactions are present, behaves very differently, i.e., the magnetization decreases at low temperatures (Figure 2(b)). This curve 
shape is typical of spin-glass and cluster-glass systems where interactions frustrate the magnetic order, giving the observed magnetization curve $[25,26]$. These interparticle interactions probably also frustrate the magnetic order of the particle surface spins, reinforcing the magnetization reduction effect.

Magnetization loops, $M(H)$, were performed at different temperatures. As an example, Figure 3(a) displays the hysteresis loops for the $\left(\mathrm{Fe}_{0.69} \mathrm{Co}_{0.31}\right) \mathrm{B}_{0.4}$ dispersed particles at three different temperatures. The different behavior of the dispersed and powder samples confirms the uniform dispersion of the nanoparticles in the polymer without dispersion of aggregates of particles. Note that the $M(H)$ cycles have a negligibly coercive field $\left(H_{C}\right)$ for $T \geq 100 \mathrm{~K}$ (see Figure 4 ). This fact is very irregular, taking into account the large irreversibility observed in the $M(T)$ curves measured under ZFC-FC conditions, which is usually associated to open magnetization cycles in the blocked regime. At low temperatures, $H_{C}$ grows abruptly, evidencing an increase of the effective anisotropy. On the other hand, the $M(H)$ behavior of the interacting particles is very different (Figure 3(b)). The powder sample shows a small $H_{C}$ in all the temperature range, smoothly increasing at low temperatures (Figure 4).

Figure 3. Magnetization loops at different temperatures for $\left(\mathrm{Fe}_{0.69} \mathrm{Co}_{0.31}\right) \mathrm{B}_{0.4}$ dispersed (a) and powder (b) particles. The insets correspond to the low field magnetization detail.

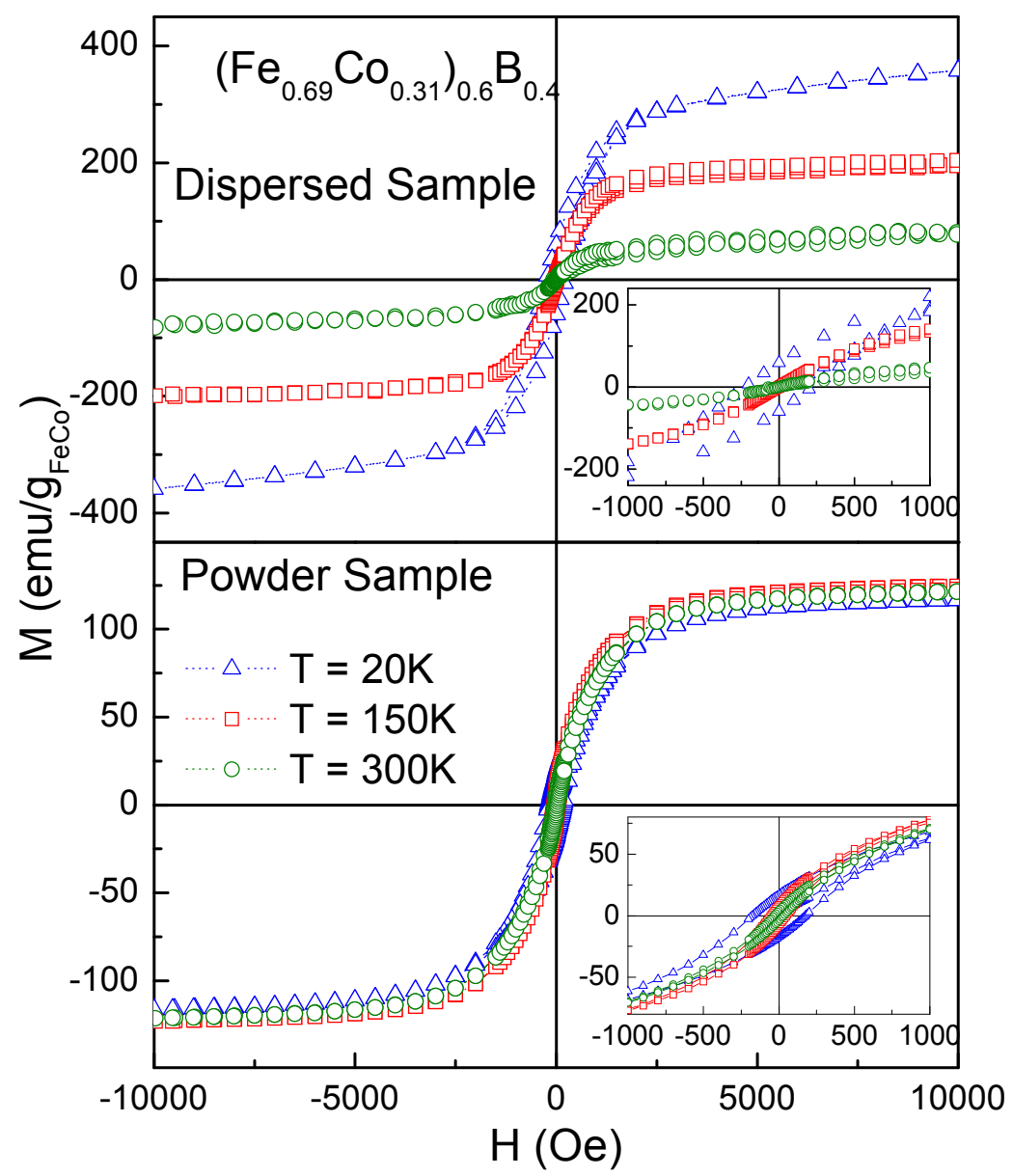


Figure 4. Coercive field $v s$. temperature for $\left(\mathrm{Fe}_{0.69} \mathrm{Co}_{0.31}\right) \mathrm{B}_{0.4}$ samples. The lines are only a guide for the eye.

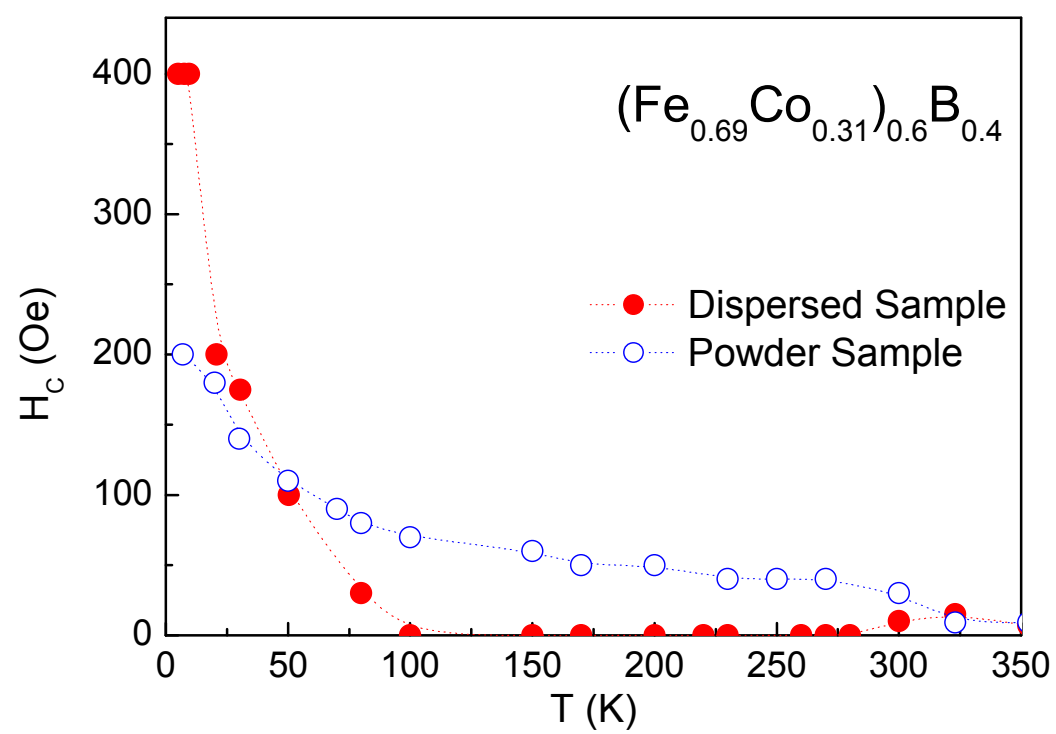

Figure 5. Saturation magnetization $\left(M_{S}\right) v s$. temperature for dispersed (non-interacting) and powder (with strong interparticle interactions) $\left(\mathrm{Fe}_{0.69} \mathrm{Co}_{0.31}\right) \mathrm{B}_{0.4}$ particles, and the bulk $\left(\mathrm{Fe}_{0.7} \mathrm{Co}_{0.3}\right)_{0.8} \mathrm{~B}_{0.2}$ and $\mathrm{Fe}_{0.7} \mathrm{Co}_{0.3}$ samples. The lines are only a guide for the eye.

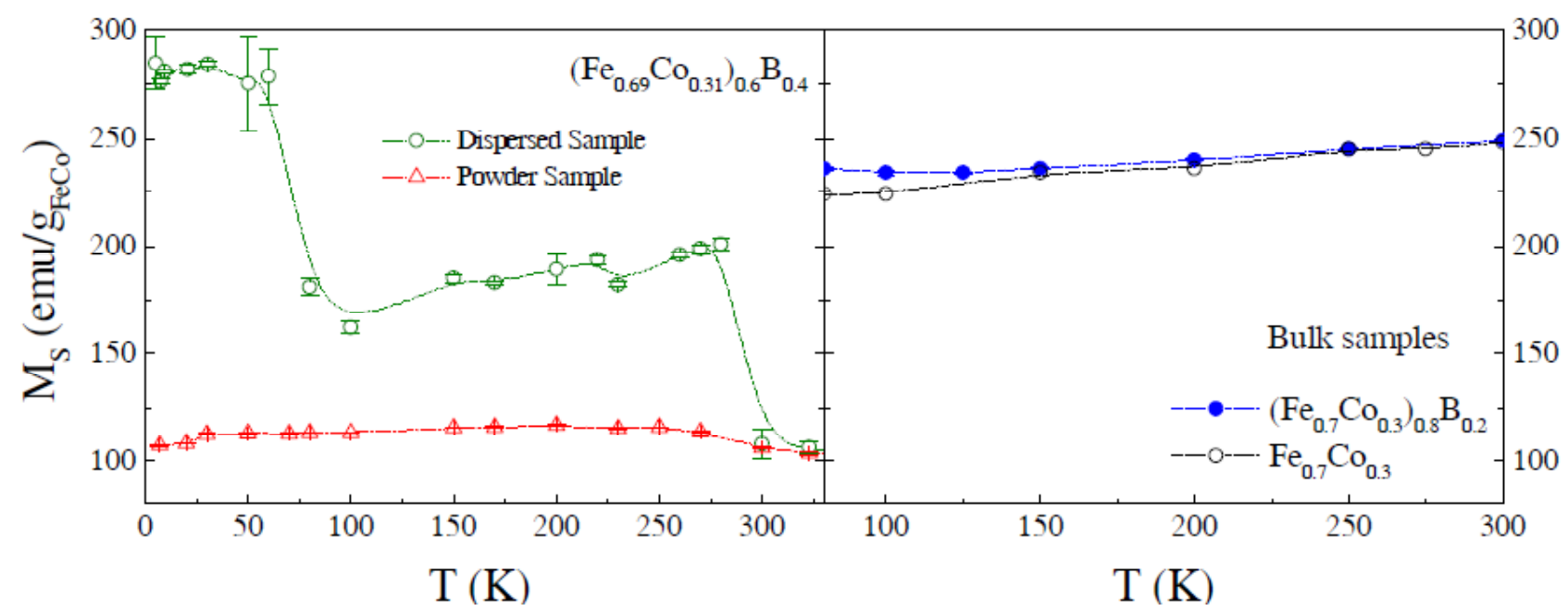

It is noticeable that $M(H)$ does not saturate even at the maximum applied field, with a linear behavior at high magnetic fields, suggesting the existence of two contributions to the magnetization: $M(H)=M_{i r r}+M_{r e v}$. The first term $\left(M_{i r r}\right)$ is related to the blocked regime, which saturates at low field; whereas the second one $\left(M_{r e v}\right)$ is related to a paramagnetic contribution to the magnetization. The saturation magnetization, $M_{S}$, of each curve is determined by subtracting the linear contribution of the magnetization at high field or, similarly, by extrapolating the magnetization at $H=0$ from the high field linear behavior. From the magnetization curves of the non-interacting system, the increase of $M_{S}$ when the temperature decreases is evident, whereas $M_{S}$ of the powder sample remains quasi-constant with a value equal to the lowest saturation magnetization measured for the non-interacting sample. $M_{S}$ as a function of temperature $(5-350 \mathrm{~K})$ curves for the dispersed and powder samples of $\left(\mathrm{Fe}_{0.69} \mathrm{Co}_{0.31}\right) \mathrm{B}_{0.4}$ are plotted particles in Figure 5. The saturation magnetization of 
the non-interacting particles presents a peculiar characteristic, showing three regions of different behaviors. In the range of temperature of $300-350 \mathrm{~K}, M_{S} \sim 100 \mathrm{emu} / \mathrm{g}_{\mathrm{FeCo}}$; in the second region $(80 \mathrm{~K}<T<280 \mathrm{~K})$ we observed a plateau of $M_{S} \sim 175 \mathrm{emu} / \mathrm{g}_{\mathrm{FeCo}}$; and in the last one $(5 \mathrm{~K}<T<70 \mathrm{~K})$ the saturation magnetization reaches $\sim 280 \mathrm{emu} / \mathrm{g}_{\mathrm{FeCo}}$. The low temperature value of $M_{S}$ is larger than the calculated value for the Fe-Co bulk alloy at this composition $(\sim 240 \mathrm{emu} / \mathrm{g})$.

In order to characterize the magnetic properties of the alloy at this composition $(x=0.69)$ and compare it with our particles, bulk samples of $\left(\mathrm{Fe}_{0.7} \mathrm{Co}_{0.3}\right)_{0.8} \mathrm{~B}_{0.2}$ and $\mathrm{Fe}_{0.7} \mathrm{Co}_{0.3}$ composition were prepared in an arc furnace. The relative boron/FeCo ratio in this sample is the maximum boron amount that the amorphous alloy can incorporate when synthesized by this method (at the eutectic point) [27]. $M(H)$ was measured at different temperatures for these bulk samples, obtaining $M_{S}$ approximately constant in the range of $5 \mathrm{~K}<T<300 \mathrm{~K}$ with a value of $M_{S} \sim 240 \mathrm{emu} / \mathrm{g}_{\mathrm{FeCo}}$ (see Figure 5) close to the pure Fe-Co alloy saturation value. The $M_{S}$ values obtained for both samples indicate that the boron content does not affect the magnetization of the system. The saturation magnetization of the bulk alloy when compared with the non-interacting nanoparticles (the dispersed sample) shows that the last one presents a very different temperature dependence, confirming that the changes in $M_{S}(T)$ observed in the particles did not originate in the intrinsic ferromagnetic order of the alloy. Consequently, the magnetization observed in the dispersed sample may evidence the existence of three different internal magnetic orders of the particle's spins.

At high temperatures, a core-shell magnetic structure of the particles is expected, i.e., a core ferromagnetically ordered, whereas the shell spins in a paramagnetic state. This magnetic disorder originates in the broken exchange bonds (which reduce the effective exchange interaction in the shell), and in the surface anisotropy, that inevitably lead to surface spin misalignment with respect to the ordered core spins $[16,17,28]$. Such spin disorder propagates from the surface to the core, giving a shell region with paramagnetic regime. In this case, the saturation magnetization measured originates in the small ordered core, also giving a negligibly small $H_{C}$ field, because it originates only in the shape anisotropy (the sample is amorphous and we assume that the particles are not spherical, rather probably having an ellipsoidal shape). From the $M_{S}$ value, an average $2 \mathrm{~nm}$ diameter for the magnetically ordered core has been estimated. At intermediate temperatures, $70 \mathrm{~K}<T<300 \mathrm{~K}$, a change in $M_{S}$ occurs, suggesting a modification of the surface contribution to the magnetization. With decreasing temperature, magnetic correlation between surface spins develops, giving rise to exchange-coupled ferromagnetic clusters in the shell, contributing to the total magnetization value. Additionally, these clusters are strongly influenced by the surface anisotropy, with the easy axis perpendicular (parallel) to the surface for the Fe (Co) spins, giving a disordered surface-spin state [18,19]. Due to the large surface anisotropy energy, the ferromagnetically ordered regions in the shell "freeze" in a disordered state, coupled to the ferromagnetic core. Because of this coupling, the freezing of the surface spins actually exerts a magnetic frustration on the core moment, giving a cluster-glass character to the whole particle, displaying anomalous closed magnetization loops with $H_{C} \sim 0$. This cluster-glass behavior avoids aligning the spins in a complete ferromagnetic order, giving the observed $M_{S}$ value. At low temperatures, $T<70 \mathrm{~K}$, an additional change in the magnetic order of the non-interacting particles is observed. The saturation magnetization value grows, evidencing the full order of the spins, yielding larger $M_{S}$ values than that measured in the bulk alloy. This effect is probably due to a modification in the conduction electron band, which is confined to metallic nanoparticles. In correspondence to the 
saturation magnetization, the coercive field shows an abrupt increase in this low temperature range. Actually, below $70 \mathrm{~K}$, the subsequent growing of the ferromagnetic regions in the shell aligned in the field direction, allowing their exchange coupling with the ferromagnetic core of the nanoparticles, making its reversal more difficult, and thus strongly increasing the effective anisotropy of the whole particle $[18,19]$. This enhancement in the magnetic order is also evidenced in the increase of the FC magnetization at low temperatures measured at $H=50$ Oe $[16,18,19]$.

An additional confirmation of the internal magnetic order change below $T=70 \mathrm{~K}$ is given by the $M(T)$ curve measured at high fields. In Figure 6 the measured $M(T)$ curve is plotted, cooling the sample down to $T=100 \mathrm{~K}$, reflecting the magnetization value originated by the internal magnetic order of the spins corresponding to this range of temperature. On the other hand, on cooling the sample down to $T=5 \mathrm{~K}$ a different magnetic order is favored, characteristic of low temperatures, where the spins are fully aligned. Performing the experiment warming the sample, the measured $M(T)$ curve has larger values than the previous experiment, corresponding to the low-temperature ordered state. This low-temperature magnetic order remains even at room temperature because it is stabilized by the applied magnetic field. These results strongly contrast with the $M_{S}(T)$ values obtained from the magnetization loops. In the loops, where a previous zero-field-cooling is performed, the internal magnetic order is frozen at each characteristic temperature and then the magnetization is recorded applying the magnetic field. In this case, the maximum applied magnetic field $H=10 \mathrm{kOe}$ is not enough to change the magnetic order at the corresponding temperature, but is still enough to stabilize the low-temperature order in the $M(T)$ experiment.

Figure 6. Magnetization vs. temperature measured, applying a field of $H=10$ kOe: cooling the sample down to $T=5 \mathrm{~K}$ with the field (open triangles), cooling the sample in zero field down to $T=100 \mathrm{~K}$ (open circles), and cooling applying the field down to $T=100 \mathrm{~K}$ (solid circles).

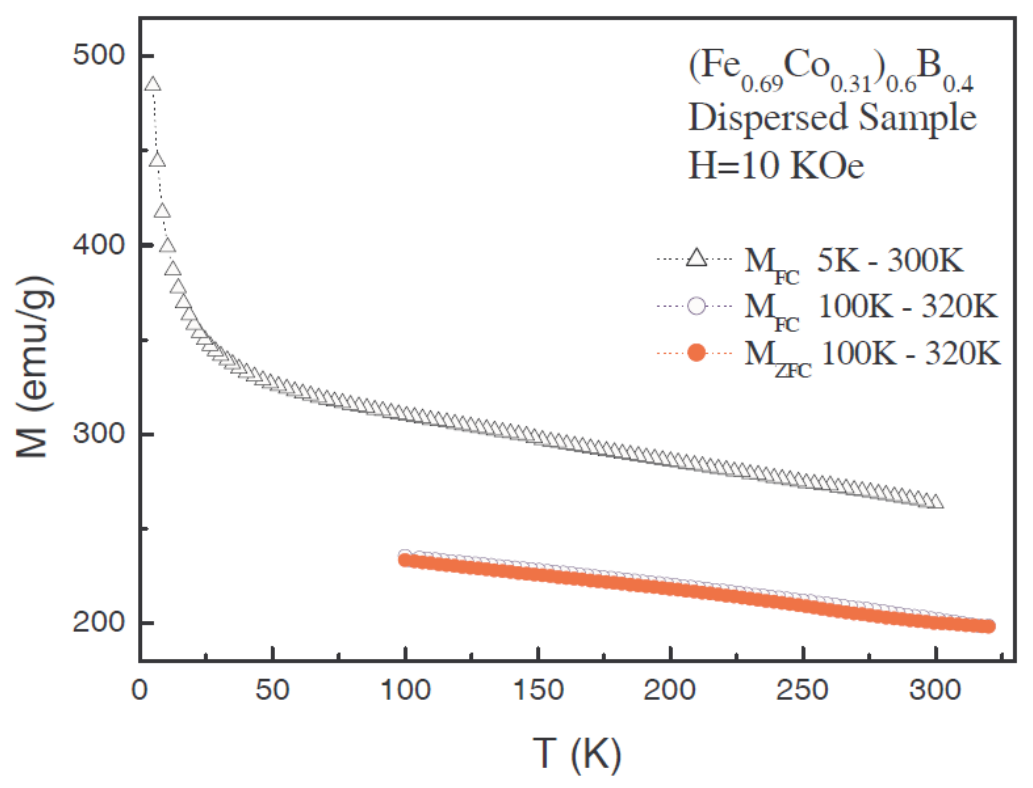


It is quite noticeable that the enhancement of the saturation magnetization decreasing temperature, related to the magnetic moment of the particles, is extremely dependent on composition. Figure 7 shows the saturation magnetization $v s$. composition for the dispersed samples at $T=300 \mathrm{~K}, 80 \mathrm{~K}$, and $5 \mathrm{~K}$ near the $x=0.69$ composition. In order to compare our results of magnetization in nanoparticles, we have also plotted the saturation magnetization for the bulk alloy. From the figure, it is evident that the magnetization enhancement takes effect only in a small range of composition, in opposition to the bulk materials where a broad maximum is observed. Moreover, magnetization increases with decreasing temperature for the nanoparticles at composition near $x=0.69$ are much larger than the changes of $M_{S}$ for the other compositions studied. Probably, in the first case, the larger magnetic moment allows the complete ferromagnetic order in each particle at low temperature, yielding these saturation magnetization values.

Figure 7. Saturation magnetization vs. composition for $\left(\mathrm{Fe}_{\mathrm{x}} \mathrm{Co}_{1-\mathrm{x}}\right)_{0.6} \mathrm{~B}_{0.4}$ dispersed nanoparticles at $T=5 \mathrm{~K}, 80 \mathrm{~K}$, and $300 \mathrm{~K}$. The dashed line is the $M_{S}$ bulk alloy value from [1]. The solid lines are only a guide to the eye.

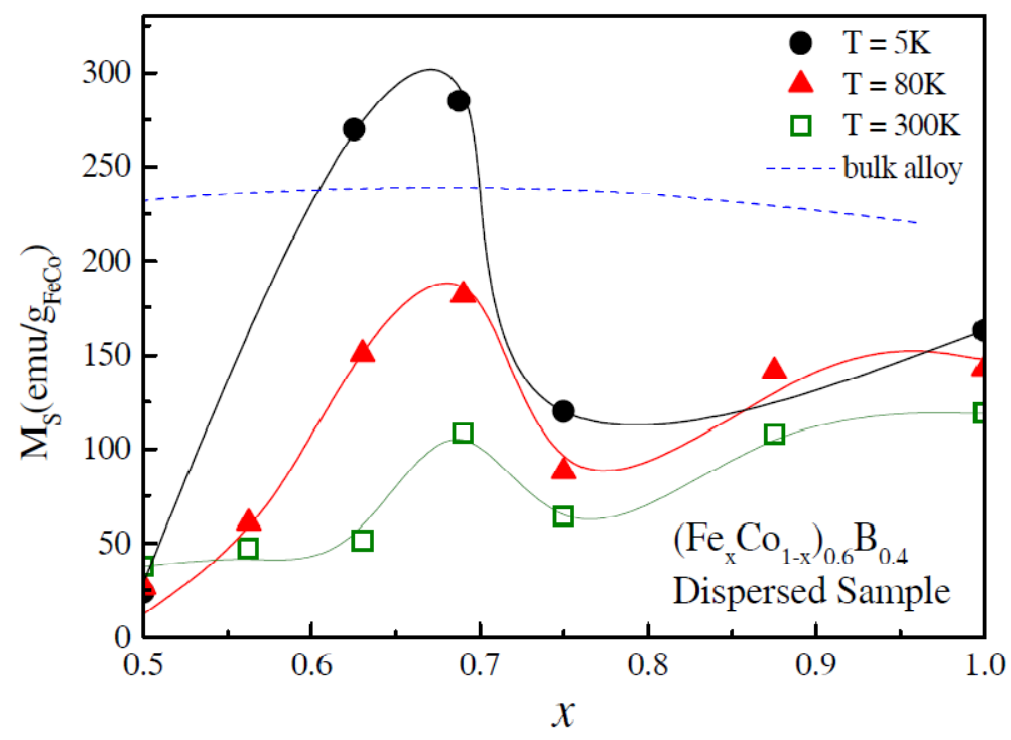

On the other hand, the powder samples have a very different behavior. As previously mentioned, the $M(T)$ measurements under $\mathrm{ZFC}$ and $\mathrm{FC}$ conditions of $\left(\mathrm{Fe}_{0.69} \mathrm{Co}_{0.31}\right) \mathrm{B}_{0.4}$ composition reveal the presence of strong interactions between particles, suggesting the possibility of frustration in the magnetic order and cluster-glass behavior. Additionally, the $M(H)$ curves are very different with respect to the non-interacting sample, confirming our description of the interparticle interaction role. The magnetization loops of the powder samples present a small but non-zero coercive field, which increases smoothly as the temperature decreases (Figures 3(b) and 4). Also, the saturation magnetization is very different from the non-interacting case: the interacting particles show a $M_{S}$ value close to the one at room temperature, approximately constant in temperature (Figure 5). The different features of both samples can be understood, considering that the interparticle interactions in the powder samples strongly affect the surface spins avoiding the growing of ferromagnetic ordered regions in the shell. In the dispersed sample case, where interparticle interactions are negligible, the spins in the shell may align along the core direction and enhance particle saturation magnetization. 
Nevertheless, intra-particle interactions are present in the "non-interacting" (dispersed) particles, suggested by the anomalous behavior of the magnetization loop shape, the coercive field and the saturation magnetization. Evidently, the contrast observed between the two samples with $\left(\mathrm{Fe}_{0.69} \mathrm{Co}_{0.31}\right) \mathrm{B}_{0.4}$ composition (where the atomic magnetic moment is a maximum) is also present in the $M_{S}$ values as a function of composition, where the maximum of $M_{S}$ in the powder sample is also observed at $x=0.69$, but this value is approximately constant in temperature.

\section{Conclusions}

We have studied the magnetic properties of $3-\mathrm{nm}\left(\mathrm{Fe}_{0.69} \mathrm{Co}_{0.31}\right){ }_{0.6} \mathrm{~B}_{0.4}$ amorphous nanoparticles, where the magnetization value $v s$. Fe/Co composition has a maximum value. Curiously, non-interacting particles (particles dispersed in a non-magnetic matrix) show very anomalous magnetization behavior. We have associated the observed results with different internal magnetic orders inside the particle with a core-shell structure, where intraparticle interactions lead to frustration in the ferromagnetic order. Interparticle interactions in the powder sample strongly affect the internal magnetic order of the spins in the particles' shell, depressing the saturation magnetization value, remaining approximately constant in temperature.

An open question remains about the low temperature value of the saturation magnetization of the non-interacting sample, which is larger than the saturation magnetization of the bulk alloy or the theoretically calculated value of $M_{S}$ for the same Fe-Co composition. We hope that this work stimulates some theoretical band calculations in order to provide an answer to this enhancement of the magnetization in a confined system.

\section{Acknowledgments}

This work was accomplished with partial support by ANCPyT, CONICET (Argentina) projects.

\section{Conflict of Interest}

The authors declare no conflict of interest.

\section{References}

1. Chikazumi, S. Physics of Magnetism; Krieger Publ. Co.: Malabar, FL, USA, 1964; Chapter 4.

2. Dorman, J.L.; Fiorani, D. Magnetic Properties of Fine Particles; North-Holland: Amsterdam, The Netherlands, 1992.

3. Hadjipanayis, G.C.; Prinz, G.A. Science and Technology of Nanostructurated Materials; Plenum Press: New York, NY, USA, 1991.

4. Fiorani, D. Surface Effects in Magnetic Nanoparticles; Springer: New York, NY, USA, 2005.

5. Kodama, R.H.; Makhlouf, S.A.; Berkowitz, A.E. Finite size effects in antiferromagnetic NiO nanoparticles. Phys. Rev. Lett. 1997, 79, 1393-1396.

6. García del Muro, M.; Batlle, X.; Labarta, A. Erasing the glassy state in magnetic fine particles. Phys. Rev. B 1999, 59, 13584-13587. 
7. Martínez, B.; Obradors, X.; Balcells, L.; Rouanet, A.; Monty, C. Low temperature surface spin-glass transition in $\gamma-\mathrm{Fe}_{2} \mathrm{O}_{3}$ nanoparticles. Phys. Rev. Lett. 1998, 80, 181-184.

8. Batlle, X.; García del Muro, M.; Labarta, A. Interaction effects and energy barrier distribution on the magnetic relaxation of nanocrystalline hexagonal ferrites. Phys. Rev. B 1997, 55, 6440-6445.

9. Dormann, J.L.; Cherkaoui, R.; Spinu, L.; Nogués, M.; Lucari, F.; D’Orazio, F.; Fiorani, D.; García, A.; Tronc, E.; Jolivet, J.P. From pure superparamagnetic regime to glass collective state of magnetic moments in $\gamma-\mathrm{Fe}_{2} \mathrm{O}_{3}$ nanoparticle assemblies. J. Magn. Magn. Mater. 1998, 187, L139-L144.

10. Mørup, S.; Tronc, E. Superparamagnetic relaxation of weakly interacting particles. Phys. Rev. Lett. 1994, 72, 3278-3281.

11. Jonsson, P.; Nordblad, P. Comment on "Erasing the glassy state in magnetic fine particles". Phys. Rev. B 2000, 62, doi: 10.1103/PhysRevB.62.1466.

12. Kodama, R.H.; Berkowitz, A.E. Atomic-scale magnetic modeling of oxide nanoparticles. Phys. Rev. B 1999, 59, 6321-6336.

13. Kachkachi, H.; Ezzir, A.; Nogués, M.; Tronc, E. Surface effects in nanoparticles: Application to maghemite $\gamma-\mathrm{Fe}_{2} \mathrm{O}_{3}$. Eur. Phys. J. B 2000, 14, 681-689.

14. Kachkachi, H.; Nogués, M.; Tronc, E.; Garanin, D.A. Finite-size versus surface effects in nanoparticles. J. Magn. Magn. Mater. 2000, 221, 158-163.

15. Iglesias, O.; Labarta, A. Finite-size and surface effects in maghemite nanoparticles: Monte Carlo simulations. Phys. Rev. B 2001, 63, doi: 10.1103/PhysRevB.63.184416.

16. De Biasi, E.; Ramos, C.A.; Zysler, R.D.; Romero, H. Large surface magnetic contribution in amorphous ferromagnetic nanoparticles. Phys. Rev. B 2002, 65, doi: 10.1103/PhysRevB.65.144416.

17. Zysler, R.D.; Romero, H.; Ramos, C.A.; de Biasi, E.; Fiorani, D. Evidence of large surface effects in Co-Ni-B amorphous nanoparticles. J. Magn. Magn. Mater. 2003, 266, 233-242.

18. De Biasi, E.; Ramos, C.A.; Zysler, R.D.; Romero, H.; Fiorani, D. Size dependence of the spin-flop transition in hematite nanoparticles. Phys. Rev. B 2005, 71, doi: 10.1103/PhysRevB.68.212408.

19. De Biasi, E.; Ramos, C.A.; Zysler, R.D.; Fiorani, D. Metropolis algorithm for simulating hysteresis in ferromagnetic nanoparticles. Phys. B 2006, 372, 345-349.

20. Halperin, W.P. Quantum size effects in metal particles. Rev. Mod. Phys. 1986, 58, 533-606.

21. Molina Concha, B.; Zysler, R.D.; Romero, H. Magnetization enhancement in Fe-Co-B alloy nanoparticles. Phys. B 2006, 384, 274-276.

22. Zysler, R.D.; Ramos, C.A.; Romero, H.; Ortega, A. Chemical synthesis and characterization of amorphous Fe-Ni-B magnetic nanoparticles. J. Mater. Sci. 2001, 36, 2291-2294.

23. Molina Concha, B.; Zysler, R.D.; Troiani, H.; Romero, H. Surface and local anisotropy effect in the magnetic order of Fe-Co-B nanoparticles. Phys. B 2004, 354, 121-124.

24. Molina Concha, B.; de Biasi, E.; Zysler, R.D. Monte Carlo simulation of Fe-Co amorphous nanoparticles magnetization. Phys. B 2008, 403, 390-393.

25. Zysler, R.D.; Fiorani, D.; Testa, A.M. Investigation of magnetic properties of interacting $\alpha-\mathrm{Fe}_{2} \mathrm{O}_{3}$ nanoparticles. J. Magn. Magn. Mater. 2001, 224, 5-11.

26. Zysler, R.D.; Ramos, C.A.; De Biasi, E.; Romero, H.; Ortega, A.; Fiorani, D. Effect of interparticle interactions in $\left(\mathrm{Fe}_{0.26} \mathrm{Ni}_{0.74}\right)_{50} \mathrm{~B}_{50}$ magnetic nanoparticles. J. Magn. Magn. Mater. 2000, 221, 37-44. 
27. Hansen, M.; Anderko, K. Constitution of Binary Alloys; McGraw Hill: New York, NY, USA, 1953.

28. De Biasi, E.; Ramos, C.A.; Zysler, R.D.; Romero, H. Magnetization enhancement at low temperature due to surface ordering in Fe-Ni-B amorphous nanoparticles. Phys. B 2002, 320, 203-205.

(C) 2012 by the authors; licensee MDPI, Basel, Switzerland. This article is an open access article distributed under the terms and conditions of the Creative Commons Attribution license (http://creativecommons.org/licenses/by/3.0/). 